\title{
Probing the Assembly of HDL Mimetic, Drug Carrying Nanoparticles Using Intrinsic Fluorescence ${ }^{\mathrm{S}]}$
}

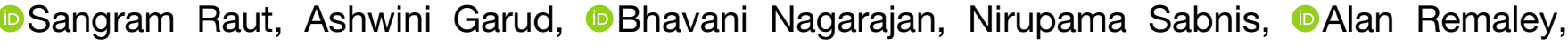 \\ Rafal Fudala, Iㅣnacy Gryczynski, @Zlygmunt Gryczynski, @ Sergei V. Dzyuba, \\ (D) Julian Borejdo, and (1)Andras Lacko
}

Departments of Physiology and Anatomy (S.R., A.G., B.N., N.S., A.L.) and Microbiology, Immunology and Genetics (R.F., I.G., Z.G., J.B.), UNT Health Science Center, Fort Worth, Texas; National Heart, Lung and Blood Institute, National Institutes of Health, Bethesda, Maryland (A.R.); and Departments of Physics and Astronomy (Z.G.) and Chemistry and Biochemistry (S.V.D.), Texas Christian University, Fort Worth, Texas

Received September 25, 2019; accepted December 13, 2019

\begin{abstract}
Reconstituted high-density lipoprotein (HDL) containing apolipoprotein A-I (Apo A-I) mimics the structure and function of endogenous (human plasma) HDL due to its function and potential therapeutic utility in atherosclerosis, cancer, neurodegenerative diseases, and inflammatory diseases. Recently, a new class of HDL mimetics has emerged, involving peptides with amino acid sequences that simulate the the primary structure of the amphipathic alpha helices within the Apo A-I protein. The findings reported in this communication were obtained using a similar amphiphilic peptide (modified via conjugation of a myristic acid residue at the amino terminal aspartic acid) that self-assembles (by itself) into nanoparticles while retaining the key features of endogenous HDL. The studies presented here involve the macromolecular assembly of the myristic acid conjugated peptide (MYR-5A) into nanomicellar structures and its characterization via steady-state and time-resolved fluorescence spectroscopy. The structural differences between the free peptide (5A) and MYR-5A conjugate were also probed, using tryptophan fluorescence, Förster resonance energy transfer (FRET), dynamic light
\end{abstract}

scattering, and gel exclusion chromatography. To our knowledge, this is the first report of a lipoprotein assembly generated from a single ingredient and without a separate lipid component. The therapeutic utility of these nanoparticles (due to their capablity to incorporate a wide range of drugs into their core region for targeted delivery) was also investigated by probing the role of the scavenger receptor type B1 in this process.

\section{SIGNIFICANCE STATEMENT}

Although lipoproteins have been considered as effective drug delivery agents, none of these nanoformulations has entered clinical trials to date. A major challenge to advancing lipoproteinbased formulations to the clinic has been the availability of a cost-effective protein or peptide constituent, needed for the assembly of the drug/lipoprotein nanocomplexes. This report of a robust, spontaneously assembling drug transport system from a single component could provide the template for a superior, targeted drug delivery strategy for therapeutics of cancer and other diseases (Counsell and Pohland, 1982).

\section{Introduction}

The physical and chemical properties of lipoproteins have been thoroughly investigated over the last several decades (Chapman, 1980). Of the different classes of circulating

This research was supported by Cancer Prevention and Research Institute of Texas (DP150091), The Rutledge Cancer Foundation, Wheels for Wellness of Fort Worth, TX, to A.L. This work was also supported in part by Texas Alzheimer's Research Care and Consortium (2018-48-51-JI) and Leukemia Texas Foundation grants to S.R. A.L. (University of North Texas Health Science Center), A.R. (National Institutes of Health) are joint contributors to the patent (US20150343069A1) on the Myr5A nanoparticles. No other conflicts are reported.

https://doi.org/10.1124/jpet.119.262899.

[S This article has supplemental material available at jpet.aspetjournals.org. lipoproteins (VLDL, IDL, LDL, and HDL), HDLs have been studied most extensively due to their antiatherogenic potential (Tall and Small, 1978; Levy, 1981; Rye et al., 2009). The athero-protective effects of HDLs have been primarily attributed to their involvement in reverse cholesterol transport (von Eckardstein et al., 2001) and their antioxidant (Soran et al., 2015), anti-inflammatory (Barter et al., 2004), and antiadhesive (Rye et al., 2009) properties. Subsequent findings revealed that most of these effects directly involve the major protein constituent of HDL, apolipoprotein A-I (Apo A-I) (Frank and Marcel, 2000; Zhang et al., 2003; Curtiss et al., 2006). Subsequently, several clinical studies evaluated the impact of synthetic/reconstituted HDL (rHDL) formulations, aiming to mediate or halt the progress of cardiovascular

ABBREVIATIONS: Apo A-I, apolipoprotein A-I; Bis-MSB, 1,4-Bis-2-methylstyryl-benzene; BSA, bovine serum albumin; CD, circular dichroism; CMC, critical micellar concentration; Dox, doxorubicin; DPH, diphenylhexatriene; EtOH, ethanol; FRET, Förster resonance energy transfer; HDL, high-density lipoprotein; KI, potassium iodide; MYR-5A, Myristoyl-5A peptide; NP, nanoparticle; rHDL, synthetic/reconstituted HDL; Rh6G, Rhodamine 6G; Val, valrubicin. 
disease (Angelin et al., 2002; Bloedon et al., 2006; Calabresi et al., 2006). Furthermore, design and characterization of rHDL nanoparticles (NPs) had been the focus of investigations (Bricarello et al., 2011; Kuai et al., 2016). The development of rHDL NPs as an approach to treat cancer as well as other diseases, including inflammatory and neurodegenerative disorders, has also accelerated over the last several years (Febbraio et al., 2001).

During the last 20 years, altered lipid metabolism has been implicated in malignant growth and metastatic tendencies (Dang, 2012; Santos and Schulze, 2012). Several studies showed that most cancers overexpress the scavenger receptor type B1 (Lacko et al., 2015; Raut et al., 2018a), a cell surface protein that is also prominently expressed by hepatocytes and by cells of hormone-producing tissues (Twiddy et al., 2012; Yuan et al., 2016; Feng et al., 2018). Patients with cancer often exhibit reduced HDL-cholesterol levels because the tumors tend to import membrane constituents from extracellular (plasma) sources (Dessì et al., 1991; Kökoğlu et al., 1994; Muntoni et al., 2009; Lee et al., 2017). The metastatic tendencies of malignant tumors have also been correlated with the overexpression of scavenger receptor type B1 and the subsequent lipid accumulation within cancer cells ( $\mathrm{Li}$ et al., 2016; Yuan et al., 2016; Panchoo and Lacko, 2018). Additional studies reported on developing synthetic/reconstituted HDL formulations, transporting payloads of anticancer agents targeting cancer cells and tumors (Shahzad et al., 2011; Sabnis et al., 2012; Rajora and Zheng, 2016; Thaxton et al., 2016; Kuai et al., 2017).

Considering the limited availability as well as associated cost of clinical grade material (Raut et al., 2018b), one of the major roadblocks of the therapeutic application(s) of rHDL or its constituents has been the requirement for injecting large doses of Apo A-I and/or rHDL NPs intravenously (Raut et al., 2018a). Consequently, smaller complexes, including those involving Apo A-I mimetic peptides, have become attractive alternatives (Navab et al., 2004; Zamanian-Daryoush and DiDonato, 2015). To conserve the lipid-binding properties of ApoA-I, the short peptides that mimic class-A amphipathic helices were designed (Anantharamaiah et al., 1985; Anantharamaiah, 1986). Initially, 18 amino acid peptides were synthesized (Anantharamaiah, 1986), followed by specific modifications of the amino acid residues (e.g., acetylation and addition of phenylalanine, etc.) to optimize the lipid binding and antiatherogenic function (Anantharamaiah et al., 2007).

Amar et al. (2010) synthesized an Apo A-I mimetic peptide (Sviridov et al., 2013) that was subsequently conjugated to a myristic acid residue via its amino terminal aspartic acid (MYR-5A). Subsequently, Lacko and Remaley, (2016) developed a versatile drug delivery agent utilizing the MYR-5A peptide (US patent: US20150343069A1). The spontaneous assembly of the MYR-5A peptide (with or without drugs) into an HDL-type nanostructure is the first report (to our knowledge) of this process to occur without additional (lipid) ingredients. Herein, we used intrinsic fluorescence of the tryptophan residues in the sequence of the peptide component as well as fluorescence of the incorporated anthracyclines to gain additional insights into the assembly process of the resultant drug transporting micellar NPs. Additional data from electron microscopy, circular dichroism, light scattering, and size exclusion chromatography have been used to support

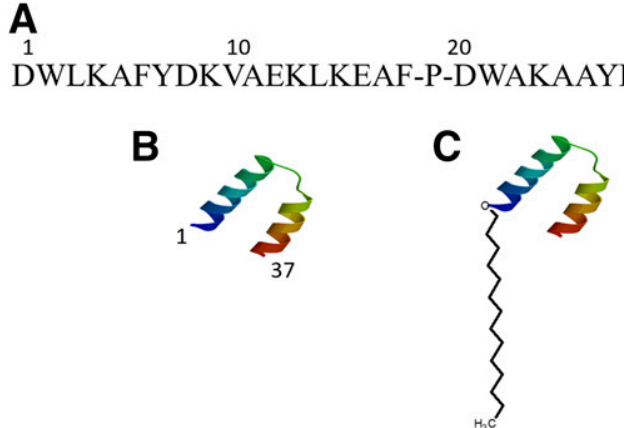

Fig. 1. (A) Amino acid sequence of 5A peptide. (B) Predicted double helical peptide structure of 5A using I-TASSER server. (C) Schematic of myristic acid-conjugated aspartic acid on $\mathrm{N}$-terminal of 5A peptide (Myr5A).

the fluorescence studies. In our view, the methodology presented here is applicable to studying structure/assembly of other types of engineered/synthetic lipoprotein nanoparticles as well as probing the basic properties of potentially important therapeutic drug carriers.

\section{Materials and Methods}

All chemicals and solvents were from commercial sources, they were of highest grade obtainable, and were used as received. The $5 \mathrm{~A}$ peptide (4218 Da) and Myr5A (4429 Da, myristic acid conjugated to 5A peptide) were prepared by Biosynthesis Inc. (Lewisville, TX). Doxorubicin (Dox) was obtained from LC Laboratories, Woburn, MA while valrubicin (Val) was purchased from China Kouting Group, China. Fluorescent dyes diphenylhexatriene (DPH), and 1,4-Bis-2-methylstyryl-benzene (Bis-MSB) as well as ethanol (EtOH) and DMSO were from Sigma Aldrich.

Chromatography and Dynamic Light Scattering. The AKTA FPLC system (Amersham Biosciences) along with Superdex-200 increase 10/300 size exclusion column (GE Healthcare) were used to analyze the MYR-5A nanoparticles by measuring the absorbance at $280 \mathrm{~nm}$. High molecular weight calibration kit containing known molecular weight proteins (MW 43,000-669,000 includes ovalbumin, conalbumin, aldolase, ferritin, thyroglobulin, and blue dextran) from GE healthcare was used to calibrate the column using $0.1 \times \mathrm{PBS}$ as the mobile phase. The particle size of 5A and MYR-5A was measured on Malvern Zetasizer ZS90 dynamic light scattering instrument.

Absorption and Fluorescence Measurements. The UV-Vis absorption spectra of tryptophan were measured with the Cary 60 UV-Vis spectrophotometer (Agilent Technologies). Fluorescence spectra of tryptophan's from 5A and MYR-5A in various solvents were measured with a Cary Eclipse fluorescence spectrometer (Varian Inc.) in square geometry setup. Both absorption and fluorescence measurement were performed using $0.4 \times 1 \mathrm{~cm}$ cuvettes with stoppers at room temperature $\left(22^{\circ} \mathrm{C}\right)$ and fluorescence was measured at magic angle conditions $\left(54.7^{\circ}\right)$. The steady-state anisotropy was measured using manual polarizers placed in excitation and emission paths. Emission spectra were measured in vertical and horizontal polarizer orientations to calculate the anisotropy (r) using following formula:

$$
r=\frac{I_{v v}-G I_{v h}}{I_{v v}-2 G I_{v h}}
$$

Where $I_{v v}$ is the emission intensity measured in parallel (vertical) orientation of both excitation and emission polarizers, $\mathrm{I}_{\mathrm{vh}}$ is emission intensity measured in perpendicular (horizontal) orientation of the emission polarizer relative to the excitation polarizer, and $G$ is instrumental correction factor.

The fluorescence lifetime of tryptophan residues, dyes, and anthracycline drugs were measured using the FluoTime-200 high-performance 


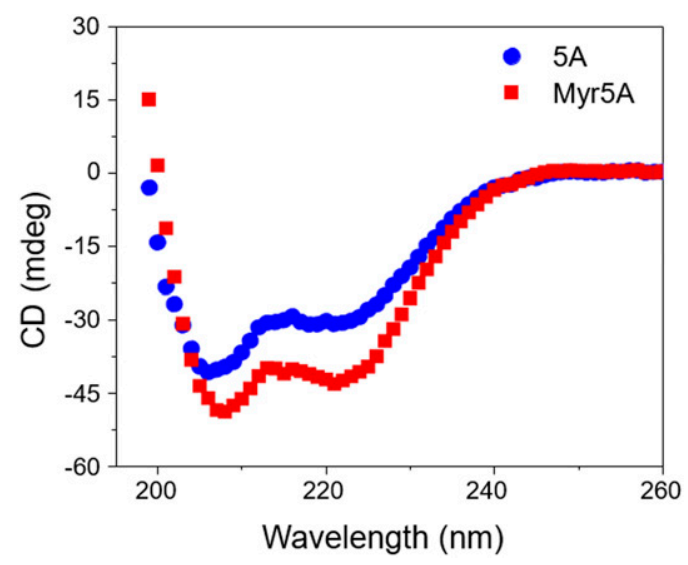

Fig. 2. Circular dichroism spectra of $5 \mathrm{~A}(5 \mathrm{mg} / \mathrm{ml})$ and Myr5A $(5 \mathrm{mg} / \mathrm{ml})$ in phosphate buffer saline at room temperature.

fluorescence lifetime spectrometer with Picoharp 300 TCSPC module (PicoQuant, GmbH). The excitation source for lifetime measurements was $290 \mathrm{~nm}$ light emitting diode with 500 picosecond pulse width. The emission of tryptophan at $350 \mathrm{~nm}$ was detected using an R3809U-50 micro-channel plate photomultiplier tube (MCPPMT; Hamamatsu, Inc). The fluorescence lifetime was measured at the magic angle condition $\left(54.7^{\circ}\right)$ to eliminate the depolarization effects due to molecular rotation. Then, the data analysis was done using FluoFit program (PicoQuant, GmbH, and Version 4.4) with a discrete multiexponential deconvolution model:

$$
\mathrm{I}(\mathrm{t})=\int_{-\infty}^{t} \operatorname{IRF}\left(t^{\prime}\right) \sum_{i} \alpha_{\mathrm{i}} e^{-(t-t) / \tau_{i}}
$$

Where IRF ( $\mathrm{t}$ ') is the instrumental response function at time $t=t$ ' and $\alpha_{i}$ is the amplitude of the $\mathrm{i}^{\text {th }}$ component decay at time $\mathrm{t}$ and $\tau_{i}$ is the lifetime of the $\mathrm{i}^{\text {th }}$ element. The average intensity and amplitude weighted lifetimes were calculated as follows:

$$
\operatorname{Avg} \tau_{I N T}=\sum f_{i} \tau_{i} \quad \text { Where, } \quad f_{i}=\frac{\sum \alpha_{i} \tau_{i}}{\sum \alpha_{i}}
$$

And

$$
\operatorname{Avg} \tau_{A m p}=\sum \alpha_{i} \tau_{i}
$$

The time-resolved fluorescence anisotropy was measured on FluoTime-200 high-performance fluorescence lifetime spectrometer with Picoharp 300 TCSPC module fitted with polarizers on excitation and emission side. Fluorescence intensity decays were measured at peak emission wavelengths (350 $\mathrm{nm}$ for tryptophan) in vertical $\left(\mathrm{I}_{\mathrm{vv}}\right)$ and horizontal $\left(\mathrm{I}_{\mathrm{vh}}\right)$ polarizer orientations that were later used to calculate and fit the anisotropy decay using FluoFit4 (Picoquant $\mathrm{GmbH}$ ) program using the following equation:

$$
r(t)=\sum_{\mathrm{i}} r_{\mathrm{i}} e^{-t / \varphi_{\mathrm{i}}}
$$

Where $r_{i}$ is the anisotropy of the $i^{\text {th }}$ component at time $t$ and $\Phi_{i}$ is the rotational correlation time of the $\mathrm{i}^{\text {th }}$ component.

The quality of the fit in the lifetime and anisotropy decay analysis was judged by the $\chi^{2}\left(\chi^{2}\right)$ value and by the quality and autocorrelation of the residuals.

Circular Dichroism Measurements. Circular dichroism (CD) spectra were acquired on a Jasco J-815. CD transitions of samples were recorded in the $260-190 \mathrm{~nm}$ range, using a $0.01-\mathrm{cm}$ demountable quartz cell. Spectra were recorded at room temperature and 1-nm resolution with a scan rate of $20 \mathrm{~nm} / \mathrm{min}$. Raw data were manipulated by subtraction of CD spectrum of the buffer. Secondary structure deconvolution was done using DichroWeb online analysis service (Whitmore and Wallace, 2004, 2008; dichroweb.cryst.bbk.ac.uk). The spectra were analyzed using CDSSTR analysis program, with reference data set (Sreerama and Woody, 2000), and the results for each secondary structure conformation were expressed as a percentage of the total.

Drug/Dye-Loaded Nanoparticles Preparation and Characterization. Anthracylines (Dox and Val) or fluorophore (DPH and Bis-MSB) loaded Myr5A micellar NPs were prepared on a microfluidic-based industry standard Nanoassemblr instrument. Myr5A (10 or $2 \mathrm{mg} / \mathrm{ml})$ and drugs/dyes $(0.25 \mathrm{mg})$ were dissolved in ethanol. PBS ( $\mathrm{pH}$ 7.4) and ethanol solutions were then injected into the different ports of the microfluidic mixer provided by Precision Nanosystems (Vancouver, BC) at 3:1 (water:ethanol) volumes, respectively, with a combined flow rate of $7 \mathrm{ml} / \mathrm{min}$. The obtained mixtures were then dialyzed against PBS overnight in 6-8 KD MWCO Spectrum Laboratories dialysis tubing to purify and remove any free drug/dye. The particle size and zeta potential of the nanoparticle is determined using Malvern Zetasizer. Moreover, transmission electron microscopy was also used to measure the particle size of nanoparticle solution drop deposited on carbon-coated formvar grids and determine the morphology of nanoparticles. One-month stability study of nanoparticles was carried out at different temperatures by measuring the size and zeta potential. Drug release study was carried out by placing the nanoparticle solution inside the dialysis bag (6-8 KD MWCO) which was placed in beaker containing $5 \%$ BSA to dissolve the released valrubicin by adsorbing onto BSA with magnetic stirring (400 rpm) at $37^{\circ} \mathrm{C}$. Samples were taken from the beaker (5\% BSA solution) to measure drug release at different time points while replacing the buffer with equal volume to maintain the sink conditions. Five percent BSA solution was used to make sure valrubicin dissolves in aqueous environment without which determination of valrubicin would be difficult.
A

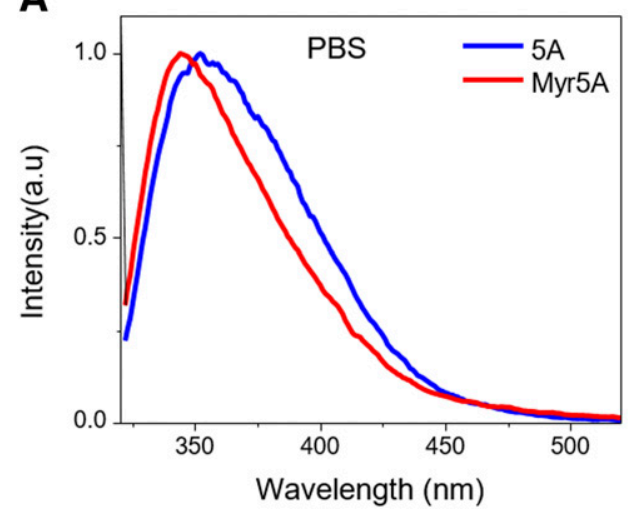

B

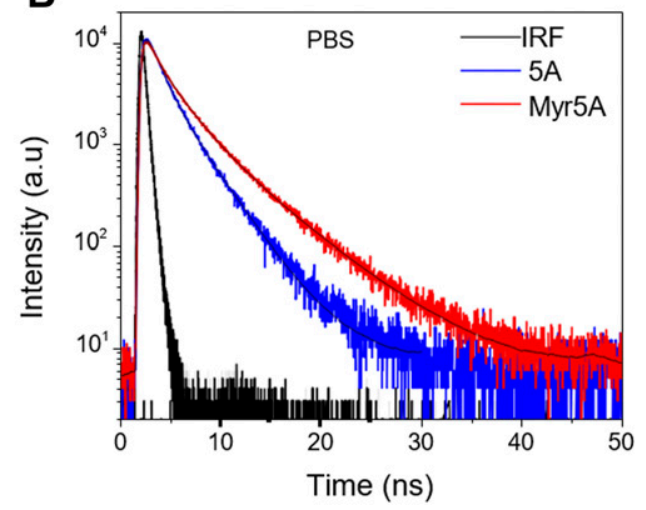

Fig. 3. (A) Emission spectra of 5A (blue) and Myr5A (red) in water (phosphate buffer saline, $\mathrm{pH} 7.4$ ) using $285 \mathrm{nmz}$ excitation. (B) Fluorescence intensity decays of $5 \mathrm{~A}$ peptide (blue) and Myr5A (red) in different solvents using excitation $290 \mathrm{~nm}$. 
TABLE 1

Recovered fluorescence lifetime parameters for 5A and Myr5A in different solvents

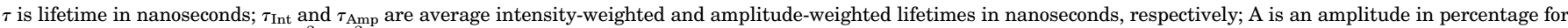
respective lifetime components; $\chi^{2}$ is $\chi^{2}$ for goodness of fit.

\begin{tabular}{|c|c|c|c|c|c|c|c|c|c|c|}
\hline \multirow{2}{*}{ Sample } & \multirow{2}{*}{ Solvent } & \multicolumn{3}{|c|}{ Components (ns) } & \multicolumn{3}{|c|}{ Amplitude (\%) } & \multirow{2}{*}{ Avg. $\tau_{\text {Int }}$} & \multirow{2}{*}{ Avg. $\tau_{\mathrm{Amp}}$} & \multirow{2}{*}{$\chi^{2}$} \\
\hline & & $\tau_{1}$ & $\tau_{2}$ & $\tau_{3}$ & $\mathrm{~A}_{1}$ & $\mathrm{~A}_{2}$ & $\mathrm{~A}_{3}$ & & & \\
\hline \multirow[t]{3}{*}{$5 \mathrm{~A}$ peptide } & PBS & $1.1 \pm 0.02$ & $2.9 \pm 0.02$ & & 62 & 38 & & 2.25 & 1.8 & 1.02 \\
\hline & Ethanol & $2.1 \pm 0.05$ & $4.3 \pm 0.03$ & & 43 & 57 & & 3.66 & 3.32 & 1.01 \\
\hline & DMSO & $6.1 \pm 0.03$ & $8.2 \pm 0.09$ & & 63 & 37 & & 6.57 & 6.47 & 0.97 \\
\hline \multirow[t]{3}{*}{$5 \mathrm{~A}$ with myristic acid } & PBS & $0.7 \pm 0.03$ & $2.4 \pm 0.02$ & $5.7 \pm 0.04$ & 40 & 44 & 16 & 3.48 & 2.22 & 0.94 \\
\hline & Ethanol & $2.0 \pm 0.03$ & $4.1 \pm 0.02$ & & 40 & 60 & & 3.56 & 3.27 & 0.99 \\
\hline & DMSO & $3.6 \pm 0.11$ & $6.6 \pm 0.03$ & & 23 & 77 & & 6.14 & 5.88 & 0.96 \\
\hline
\end{tabular}

Quenching Study. Potassium iodide (KI) was used as quencher in this study. Increasing concentrations $(0-200 \mathrm{mM})$ of KI in PBS were prepared, to which $50 \mu \mathrm{l}$ of drug/dye loaded MYR-5A NPs were added to measure the florescence of tryptophan. Fluorescence intensity of drug/dye was measured using Varian Cary 60 fluorimeter and data were analyzed to study the quencher accessibility to tryptophan in the peptide sequence.

\section{Results}

The predicted conformation of the 5A peptide was obtained by using I-TASSER server (Roy et al., 2010; Yang et al., 2015) and exhibited a "helix-loop-helix" structure (Fig. 1B). The ApoA 1 mimetic 5A peptide is composed of 37 amino acids with proline (P) at position \#19 (Fig. 1A). This structure was modified by incorporating a myristic acid residue via attachment through the N-terminal aspartic acid (D) to yield an amphipathic molecule composed of a hydrophilic (peptide) and hydrophobic (myristic acid) moieties, MYR-5A (Fig. 1C). To check if there is any change in the secondary structure of $5 \mathrm{~A}$ as the result of myristic acid conjugation, $\mathrm{CD}$ measurements were done, which indicated that both $5 \mathrm{~A}$ and MYR-5A contained comparable amounts of $\alpha$-helical $(\sim 40 \%)$ and unordered structures (Fig. 2). Additional concentrationdependent studies did not show significant changes in secondary structure between 5A and MYR-5A (Supplemental Fig. 1).

Considering the structure of Myr5A and the fact that it is soluble in water (phosphate buffered saline, $\mathrm{pH}$ 7.4), even at high concentrations (i.e., $30 \mathrm{mg} / \mathrm{ml}$ ), we hypothesized that MYR-5A could form micellar structures in aqueous environment, although it was likely to exist as a monomer in organic solvents such as EtOH and DMSO. To further characterize the properties of the MYR-5A as compared with those of $5 \mathrm{~A}$, photo-physical studies were carried out using the responses of two tryptophan residues (Trp 2 and $\operatorname{Trp} 21)$ located in the 5A sequence.

The MYR-5A peptide sequence contains all three intrinsically fluorescent amino acids; tryptophan (W), tyrosine (Y), and phenylalanine (F). To simplify the approach used during these studies, tryptophan was selectively excited at $290 \mathrm{~nm}$ for all fluorescence studies. Initially, the tryptophan emission of both 5A and MYR-5A were investigated in different solvents (Supplemental Fig. 1). However, in water, an approximately 7-nm blue shift was observed (Fig. 3A) for the MYR-5A emission peak $\left(\lambda_{\mathrm{Ems}}: 345 \mathrm{~nm}\right)$ compared with the free $5 \mathrm{~A}\left(\lambda_{\mathrm{Ems}}: 352 \mathrm{~nm}\right)$ peptide. It appeared that in EtOH and in DMSO both (free and MYR conjugated) peptides existed as monomers because no changes in emission peak ( $\lambda$ Ems: $\sim 345 \mathrm{~nm}$ ) were observed, suggesting that the solvent accessibilities of both tryptophan residues were similar (Supplemental Fig. 2), thus suggesting that one or both of the tryptophans (of the MYR-5A peptide) were buried deep in the nonpolar region of its secondary/ tertiary structure. This indicates that the average environments surrounding the respective tryptophan residues were similar. This observation may be ascribed to the solvatochromic nature of one of the tryptophan residues (Trp 2: close to $\mathrm{N}$-terminal end) that could be buried in the interior of the MYR-5A micellar structure and thus would lack solvent access. These findings were subsequently validated using timeresolved fluorescence measurements (Fig. 3B) and comparing the fluorescence intensity decays. The recovered parameters for fluorescence lifetime measurements for free $5 \mathrm{~A}$ peptide and MYR-5A in three different solvents (Table 1). In EtOH and DMSO, no major changes in fluorescence lifetimes were noted (intensity decays presented in Supplemental Fig. 3). On the other hand, in line with our earlier observations regarding the
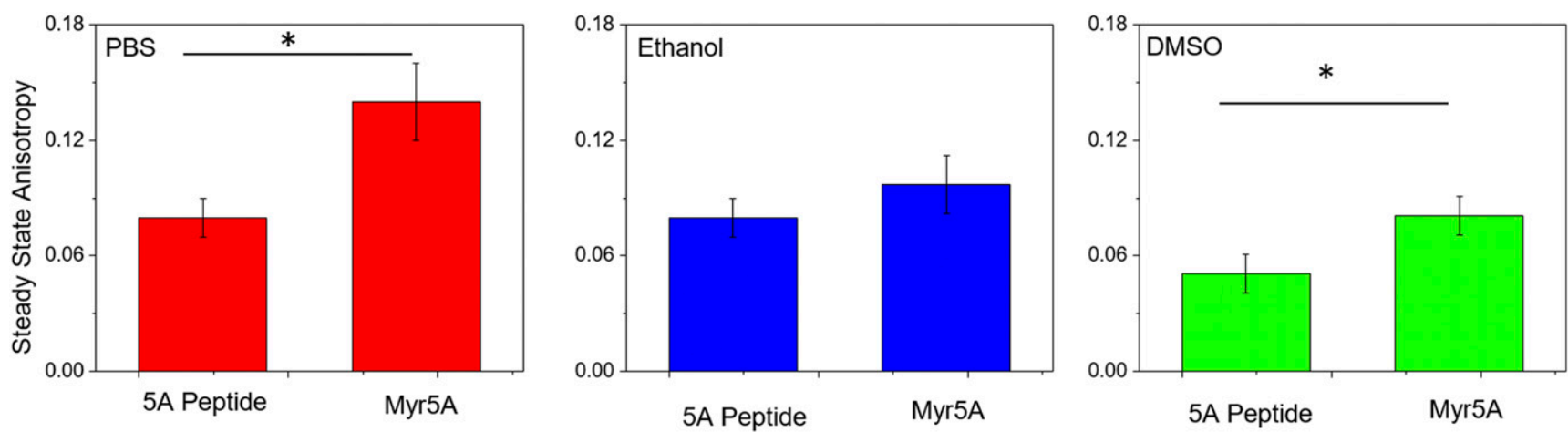

Fig. 4. Steady-state emission anisotropy of tryptophan in $5 \mathrm{~A}$ and Myr5A in different solvents observed at $350 \mathrm{~nm}$. Ex $290 \mathrm{~nm}$. ${ }^{*} P<0.05$. 
TABLE 2

Recovered anisotropy decay parameters for 5A and Myr5A in different solvents

$\mathrm{R}_{0}$ is anisotropy at time zero, $\theta$ is a rotational correlation time in nanoseconds, $\mathrm{A}$ is an amplitude associated with respective rotational correlation time, SS $\mathrm{r}$ is steady-state anisotropy, $\chi^{2}$ is $\chi^{2}$ for goodness of fit.

\begin{tabular}{|c|c|c|c|c|c|c|c|c|}
\hline \multirow{2}{*}{ Sample } & \multirow{2}{*}{ Solvent } & \multirow{2}{*}{$\mathrm{R}_{0}$} & \multicolumn{2}{|c|}{ Components (ns) } & \multicolumn{2}{|c|}{ Amplitude (\%) } & \multirow{2}{*}{$\mathrm{SS} \mathrm{r}$} & \multirow{2}{*}{$\chi^{2}$} \\
\hline & & & $\Theta_{1}$ & $\Theta_{2}$ & $\mathrm{~A}_{1}$ & $\mathrm{~A}_{2}$ & & \\
\hline \multirow[t]{3}{*}{ 5A peptide } & Water & 0.25 & 1.07 & 25.6 & 86 & 14 & 0.082 & 1.07 \\
\hline & Ethanol & 0.24 & 1.34 & 9.19 & 71 & 29 & 0.083 & 1.24 \\
\hline & DMSO & 0.24 & 1.16 & 16.23 & 85 & 15 & 0.051 & 1.18 \\
\hline \multirow{3}{*}{ 5A with myristic acid } & Water & 0.26 & 1.31 & 22.00 & 41 & 59 & 0.16 & 0.94 \\
\hline & Ethanol & 0.24 & 1.81 & 12.52 & 74 & 26 & 0.097 & 1.31 \\
\hline & DMSO & 0.25 & 1.54 & 8.86 & 63 & 37 & 0.084 & 1.06 \\
\hline
\end{tabular}

fluorescence emission peak shift, we observed the presence of a long lifetime component (5.7 nanoseconds) for the MYR-5A complex in PBS (aqueous solvent). The average lifetimes for the MYR-5A (3.48 nanoseconds) complex was significantly longer than that of the $5 \mathrm{~A}$ ( 2.25 nanoseconds) in water. These results suggested that MYR-5A formed micellar structures in water due to its solubility and lack of solvent access to tryptophan residues.

To confirm the micellar assembly, we measured the steadystate anisotropy of tryptophan from both the 5A peptide and the MYR-5A complex (Fig. 4). In an aqueous environment, The MYR-5A monomer appeared to assemble into larger micellar structures based on significantly higher anisotropy ( 0.13 vs. 0.07) compared with that observed for the $5 \mathrm{~A}$ peptide. In EtOH and DMSO, the changes in anisotropy were significantly smaller. Furthermore, time-resolved anisotropy of $5 \mathrm{~A}$ and Myr-5A in different solvents indicated their respective different behaviors (decays not shown). The recovered parameters for time-resolved anisotropy measurements were assessed (Table 2). It is evident that the $r_{0}$ value $(0.26>0.25)$ and the amplitude $(\sim 59 \%>\sim 14 \%)$ associated with longer correlation times were higher for the MYR-5A as compared with the $5 \mathrm{~A}$ peptide. In contrast, the MYR-5A anisotropy decays in both EtOH and DMSO did not show appreciable changes in correlation times compared with 5A (anisotropy decays presented in Supplemental Fig. 4). Small increases in the amplitude values for longer correlation times in $\mathrm{EtOH}$ and DMSO might be ascribed to the presence of the aminoterminal attached myristic acid residue.

Next, we determined the critical micellar concentration (CMC) of MYR-5A in aqueous buffer system using a fluorescent dye Rhodamine 6G (Rh6G) by measuring change in fluorescence anisotropy. Figure 5 left panel shows the steady-state anisotropy of Rh6G for different concentrations of MYR-5A. It shows definite increase in fluorescence anisotropy of Rh6G after about $500 \mu \mathrm{M}$ MYR-5A concentration, suggesting the initiation of micellar assembly. The CMC of MYR-5A was determined to be $900 \mu \mathrm{M}$. This was further validated by measuring the Rh6G time-resolved anisotropy below and above CMC of MYR-5A as shown in Fig. 5 right panel. The fast anisotropy decay of free Rh6G and Rh6G in $100 \mu \mathrm{M}$ MYR$5 \mathrm{~A}$ are identical, showing no interaction among the dye and MYR-5A molecules. However, Rh6G in $7500 \mu \mathrm{M}$ MYR-5A shows slow anisotropy decay $\left[\theta_{1}: 0.62\right.$ nanoseconds $(12 \%), \theta_{2}$ : 18 nanoseconds (88\%)], suggesting encapsulation of Rh6G in large nanoparticle structures with long rotational correlation time. Further studies are needed to ascertain the effects of other environmental parameters (e.g., salt concentration, temperature, and $\mathrm{pH}$ ) on the assembly of MYR-5A nanoparticles.

To estimate the size of the micellar structures, we performed fast protein liquid chromatography studies on empty MYR-5A nanoparticles. A standard curve was constructed using a high molecular weight protein calibration kit (Fig. 6A). The MYR-5A sample showed a single narrow peak on Superdex 200 size exclusion column, suggesting formation of uniform monodisperse nanoparticles. Based on the elution profile and the standard curve, an average molecular weight for these micellar structures calculated to be $\sim 75,000$ (Fig. 6B), corresponding to the approximate size of pre-beta HDL. The number of individual MYR-5A molecules in each micelle was estimated to be $\sim 17$. The size and number of molecules per micelle may change based upon the salt concentration, $\mathrm{pH}$, and temperature.

Because micellar structures are routinely used for drug delivery applications, we investigated the encapsulation of
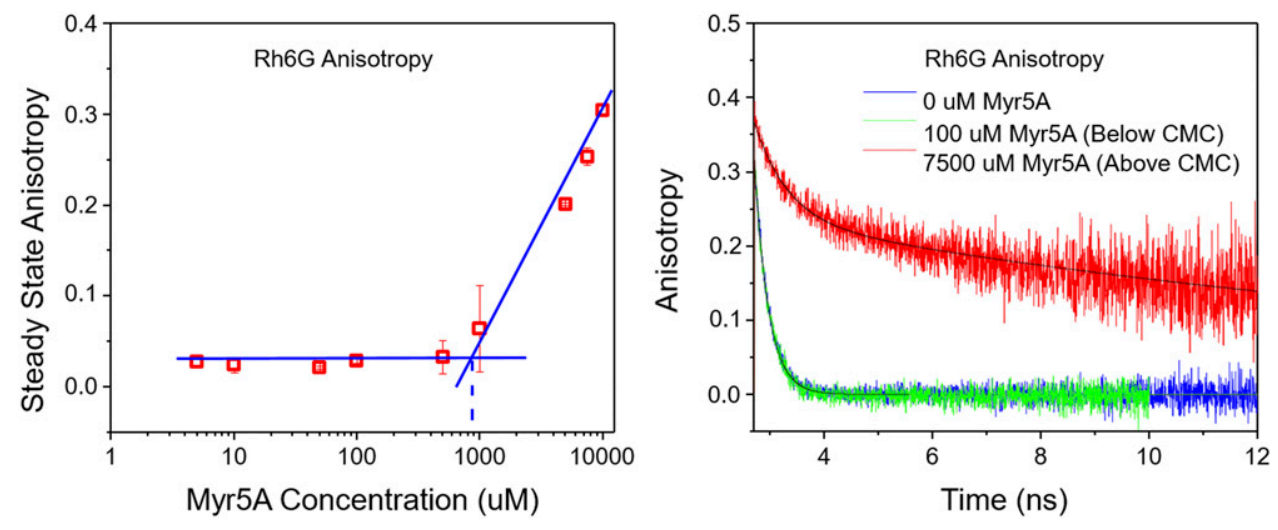

Fig. 5. Left panel: Determination of critical micellar concentration of Myr5A using Rh6G steady-state fluorescence anisotropy. Right panel: Time-resolved anisotropy of Rh6G at different concentrations (blue line: free Rh6G or no Myr5A, green line: Rh6G in $100 \mu \mathrm{M}$ Myr5A, which is below CMC, red Line: Rh6G in $7500 \mu \mathrm{M}$ Myr5A, which is above CMC) of Myr5A in PBS at pH7.4. 

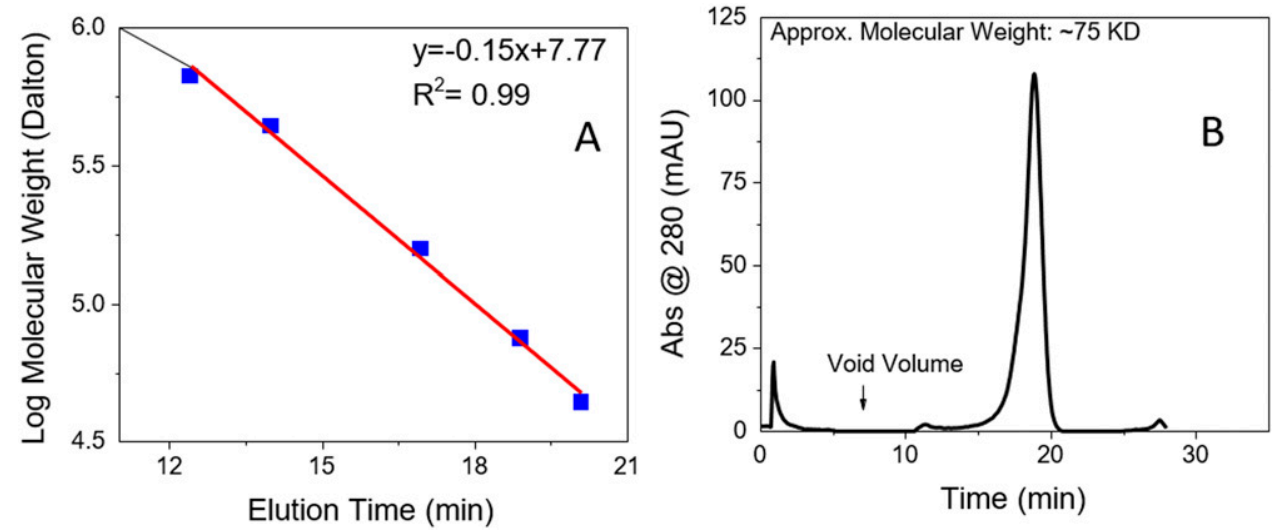

Fig. 6. (A) Calibration curve for Superdex 200 increase 10/300 column using known molecular weight proteins. (B) Fast protein liquid chromatography elution profile of Myr5A. a model cargo, specifically a classic hydrophobic fluorophore Bis-MSB. Selected molar concentrations of Bis-MSB were used to make "drug loaded" MYR-5A NPs. Fluorescence lifetime of the tryptophan residues was monitored as tryptophan and Bis-MSB are known to form a suitable FRET pair with $R_{0}$ value of $32.15 \AA$ (Fig. 7A). In case of DMSO in which MYR-5A was shown to exist in a monomeric form, no significant changes in fluorescence lifetime of the donor (tryptophan) were observed (Supplemental Fig. 5; Supplemental Table 1), which suggested lack of energy transfer. On the other hand, in a water-based solvent (PBS), there was a significant change in donor lifetime upon increasing the concentration of Bis-MSB (Fig. 7B; Supplemental Table 2). The FRET efficiencies were $34.5 \%, 61.8 \%$, and $82.7 \%$ for $100: 1,50: 1$, and $25: 1$ molar ratios of Myr5A:Bis-MSB, respectively. These findings suggested a close proximity of the encapsulated fluorescent cargo to tryptophan residues inside these small NPs.

To test the feasibility of encapsulating therapeutically relevant payloads, we used three different compounds with distinct physicochemical properties (hydrophobicity parameters). Dox, Val, and Diphenyl hexatriene (DPH) were used as model therapeutic cargos with increasing $\log \mathrm{P}$ (hydropobicity) values to produce nanoparticle assemblies. The encapsulation following nanoparticle assembly, and purification was confirmed by measuring the steady-state anisotropy of the three selected molecules (Fig. 8A), which was statistically significant compared with free drugs/dyes. To determine the relative position inside Myr5A complex of these compounds, fluorescence quenching studies using KI were carried out
(Fig. 8B). The emission of Dox was quenched to the largest extent, whereas that of DPH was the least quenched. These observations along with literature data (mentioned below) were used to approximate the location of each of these molecules (Fig. 8C). Dox, being an amphipathic molecule (and considerably more hydrophilic compared with the two other compounds, was expected to be repelled by the hydrophobic myristic acid carbon chain, indicating its likely position in the vicinity of the amino acid residues in the peptide sequence where solvent and KI can access it. Val, which is bearing a side chain attached to anthraquinone, might reside at the interface of the peptide and the myristic acid and thus may be buried deeper in the micellar structure (moderate quenching). Previous studies on anthracycline encapsulation in large lipid vesicles showed that dihydroxyanthraquinone moiety of the anthracycline is known to be excluded from the phospholipid carbon chains and exist near polar head group exposed partially to water (Gallois et al., 1996, 1998). Our quenching studies lead to the same conclusions. Moreover, DPH being highly hydrophobic and a well-known lipid probe (Lentz, 1989) would likely occupy the core region of hydrophobic tails of the MYR-5A micellar nanoparticles and would have little or no access to the solvent and KI. However, we did see a small $(<10 \%)$ amount of quenching of $\mathrm{DPH}$, which may be ascribed to either long-range electron transfer (Zelent et al., 1996) or DPH bound to hydrophobic residues in the peptide structure. Although preliminary in nature, these findings suggest a process whereby drug candidates may be evaluated for efficient incorporation into MYR-5A micellar NP
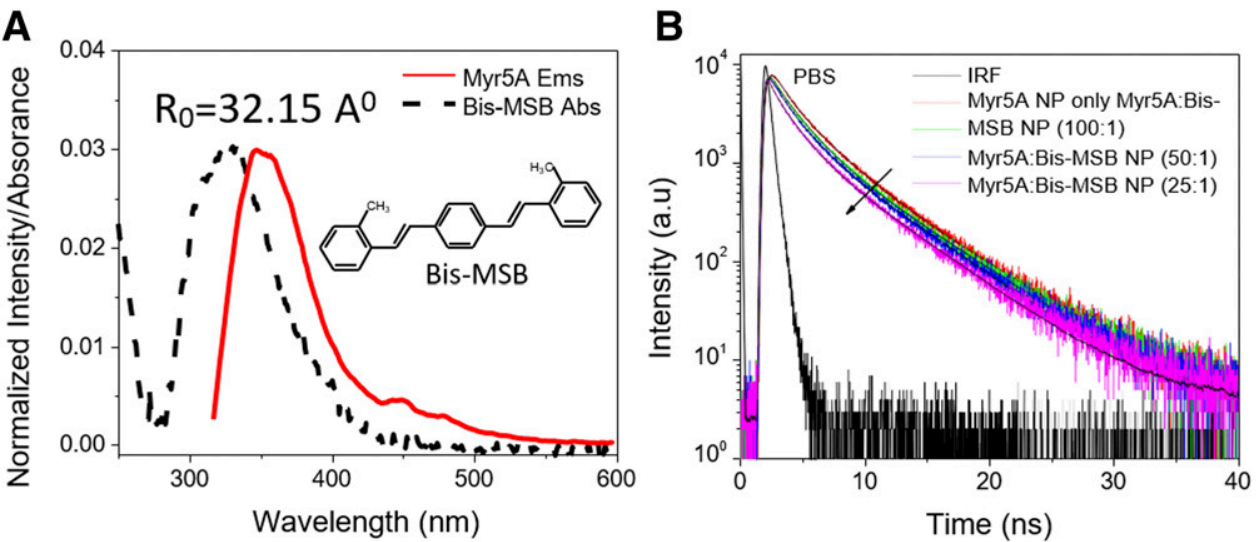

Fig. 7. (A) Spectral overlap of Bis-MSB absorbance and tryptophan emission. (B) Donor (Trp) lifetime in presence of acceptor (Bis-MSB) in DMSO. (C) Donor (Trp) lifetime in presence of acceptor (Bis-MSB) in Water/PBS.Trp, Tryptophan. 
TABLE 3

Recovered parameters from dynamic light-scattering measurements for particle size

\begin{tabular}{lcccc}
\hline \multirow{2}{*}{ Sample } & \multicolumn{4}{c}{ Diameter (nm) } \\
\cline { 2 - 5 } & Intensity Weighted & Volume Weighted & Number Weighted & PDI \\
\hline Myr5A & $7.2 \pm 2.0$ & $5.7 \pm 1.7$ & $6.6 \pm 1.2$ & 0.13 \\
$5 \mathrm{~A}$ & $3.3 \pm 0.42$ & $3.2 \pm 0.40$ & $3.3 \pm 0.25$ & 0.10 \\
\hline
\end{tabular}

PDI, polydispersity index.

transporters, based on their respective $\log P$ values. We see/ envision potential application in encapsulating drug combinations with differing $\log P$ values. Further experimental and computational studies may be needed to precisely predict the effects of $\log P$ values on the location and incorporation efficiencies of specific therapeutic payloads into the MYR$5 \mathrm{~A} \mathrm{NPs.}$

We also measured the size, drug release, encapsulation efficiency, and stability of drug-loaded Myr5A nanoparticles (Table 3). Figure 9A shows the elution profiles of the MYR-5A nanoparticles with different payloads. It is observed that drug/ dye-loaded nanoparticles were larger in size compared with free MYR-5A, whereas the elution profiles on size exclusion chromatography (column specs) showed about the same elution times. Figure 9B shows the representative TEM image of Val loaded MYR-5A nanoparticles. The average size was estimated using ImageJ size estimation protocol and found to be $50 \pm 10 \mathrm{~nm}$ (more than 300 individual nanoparticles were included in the measurement). Drug release from these nanoparticles was found to be slow (close to zero order) over the period of 72 hours (Fig. 9C). Drug encapsulation using two different anthracyclines (doxorubicin and valrubicin) was found to be different owing to their different physical properties. Figure 9D shows the more hydrophobic Val with high encapsulation efficiency at $15 \mathrm{mg} / \mathrm{ml}$ MYR-5A concentration, whereas for Dox to achieve comparable encapsulation, $30 \mathrm{mg} /$ ml MYR-5A was needed. For the stability studies, we incubated Val-loaded MYR-5A nanoparticles at different temperatures and monitored their size and zeta potential over the period of 1 month. Supplemental Table 3 shows the results of these studies. Based on these findings, it is evident that MYR-5A nanoparticles are very stable not only at $-20^{\circ} \mathrm{C}$ and $4^{\circ} \mathrm{C}$ but even at room temperature $\left(22^{\circ} \mathrm{C}\right)$. The polydispersity index also reflected the homogeneity of the preparation. The hydrodynamic size of Val-loaded MYR-5A nanoparticles is larger than observed in TEM, which is commonly observed for nanoparticles. Thus, hydrophobic payloads are ideally suited for these MYR-5A lipoprotein nanoparticles and could find widespread applications in the treatment of cancer as well as neurodegenerative and inflammatory diseases.

\section{Discussion}

Numerous micellar nanoparticles have been developed for drug delivery due to their small size, ability to incorporate multiple payloads, ease of manufacturing, and their ability to target specific tissues (Navab et al., 2004; Kuai et al., 2016, 2017; Raut et al., 2018). In addition, a great deal of research has been done on self-assembly of block copolymers and their use in drug delivery research. However, lipo-peptide-based micelles for drug delivery is a relatively novel concept considering the lack of publications in this area. In the present work, we report the design and characterization of actively targeted micelles using an amphipathic helical peptide, conjugated to a fatty acid molecule. We hypothesize that the assembly of the MYR-5A micelles is very similar to that of the di-block copolymers and is governed by the affinity (or repulsion) of the specific components of the MYR-5A molecules toward the aqueous environment. The core formed from the cluster of myristic acid residues is protected from water by the hydrophilic peptide shell on the surface, resulting in the specific size and morphology of these structures as driven by thermodynamic forces. The MYR-5A molecule must be present in sufficient quantity/critical concentration in solution to self-assemble into micelles. However, when we compare the
A

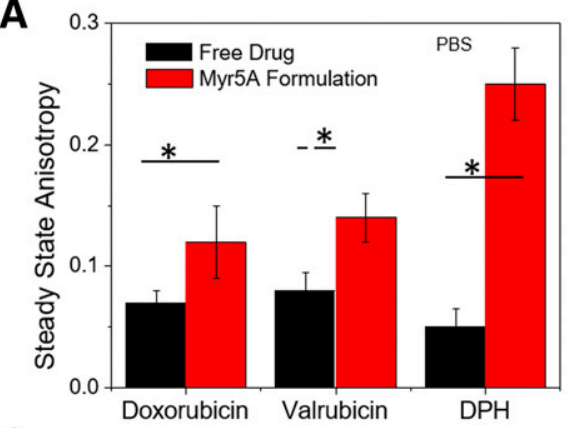

C

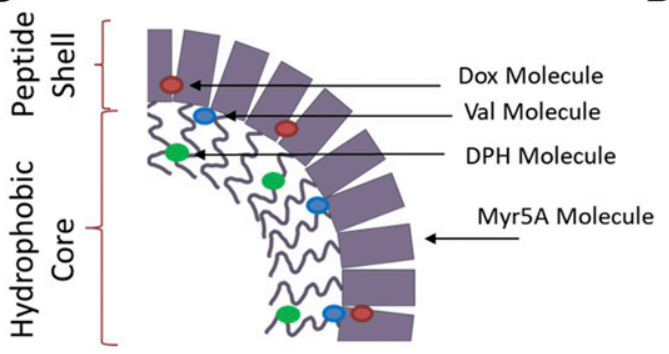

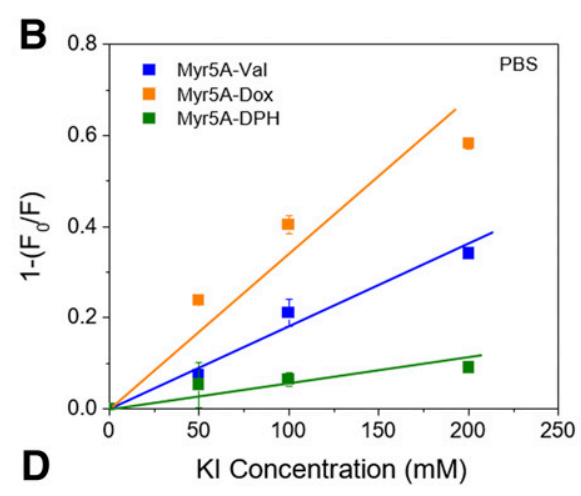

\begin{tabular}{lc}
\hline \multicolumn{1}{c}{ Compound } & Log $\mathbf{P}$ \\
\hline Doxorubicin (Dox) & 1.3 \\
Valrubicin (Val) & 4.0 \\
$\begin{array}{l}\text { Diphenyl Hexatriene } \\
\text { (DPH) }\end{array}$ & 6.1 \\
\hline
\end{tabular}

Fig. 8. (A) Steady-state anisotropy of free and encapsulated drug in Myr5A nanoparticles. (B) Stern-Volmer quenching data using $\mathrm{KI}$ as a quencher. (C) Schematic showing approximation of location of each encapsulated compound in Myr5A nanoparticle. (D) Table showing Log $\mathrm{P}$ (taken from Pubchem) values of each compound used in the experiment. $* P<$ 0.05 . 

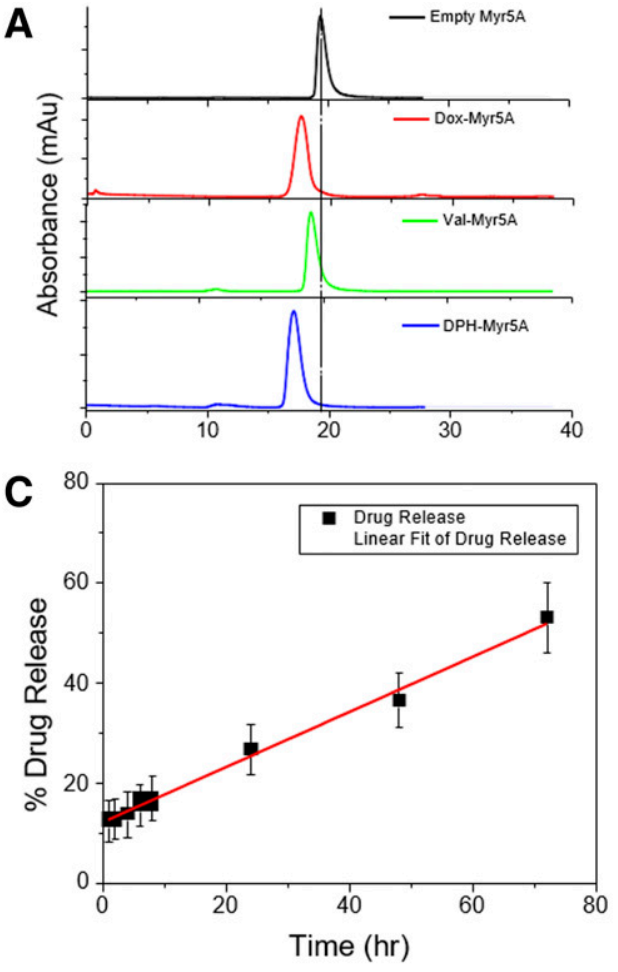

B
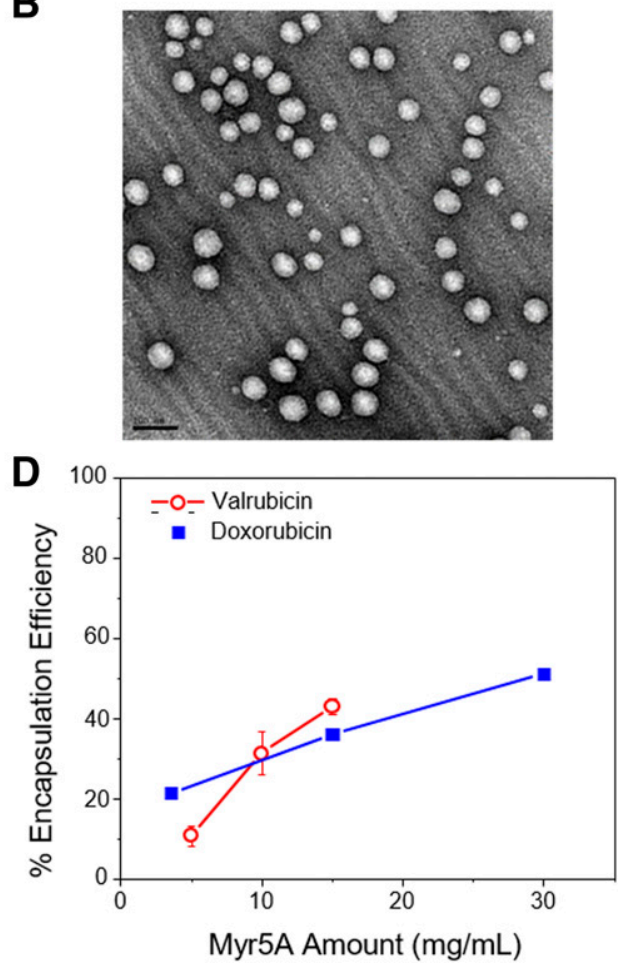

Fig. 9. (A) Elution profiles of empty, Dox, Val, and DPH Myr5A nanoparticles on size exclusion column. (B) TEM image of valrubicin loaded Myr5A nanoparticles, scale bar, $100 \mathrm{~nm}$, average size: $50 \pm 10$ $\mathrm{nm}$. (C) Drug release profile of valrubicin from Myr5A nanoparticles. (D) Encapsulation efficiency of Dox and Val at different Myr5A concentrations.
CMC of the lipopeptide with other commonly used surfactants/ detergents like SDS (8.2 mM), the CMC of MYR-5A is considerably lower. Similarly, block copolymers tend to have much lower CMC (an order of magnitude lower compared with detergents) a favorable property for invivo applications.

During these studies we have also found that several different types of (drug) molecules can be encapsulated into the core of the MYR-5A nanoparticles. The encapsulation efficiency/loading and location of the molecule generally depend on the physico-chemical properties of the given compound to be encapsulated. Moreover, it is anticipated that hydrophilic molecules will have lower loading tendencies $(<10 \% \mathrm{w})$ in such micelles compared with hydrophobic drugs. The location of the molecule in the interior (core) of the micellar structure will also likely influence its release into media (plasma or buffer). Hydrophilic drugs entrapped in the shell of the micelles would have fast or burst release profile, whereas drugs encapsulated inside the core will be released slowly as is the case for Val (Fig. 9). In case of targeted micellar preparations, the slow drug release behavior may not be relevant because the drug will be released into the cytoplasm of the target cell as once the nanoparticle is bound to a receptor. Hence, it is expected that for targeted preparations like MYR-5A, most of the drug will be delivered in first few hours rather than at later time points.

The potential toxicity of the payload delivered via these micellar nanoparticles are of importance because undesirable off-target effects, including eruption of palmar-planter skin and cardiac toxicity associated with from doxorubicin therapy, have been observed (Lorusso et al., 2007). In the case of MYR$5 \mathrm{~A}$, there are several clinical studies using the $5 \mathrm{~A}$ peptide component reporting its safe administration into cynomolgus monkey (Bourdi et al., 2018). No hematologic or organ toxicities were observed during this study. It is anticipated that the MYR-5A/drug formulations will behave similarly.

\section{Conclusions}

In summary, we evaluated the spectroscopic properties of 5A and MYR-5A peptides to gain structural insights about the assembly of MYR-5A micellar NPs. In our view, this is the first report of lipoprotein-type assemblies accomplished without a separate lipid component as one of the starting materials. The structural aspects of MYR-5A were further elucidated using size measurements and size exclusion studies. A combination of FRET and fluorescence-quenching studies provided further insights into the payload incorporation processes. Drug release, encapsulation efficiency, and stability data suggested a rational design for drug-NP complexes for therapeutic delivery and related applications.

\section{Authorship Contributions}

Participated in research design: Raut, Remaley, Lacko.

Conducted experiments: Raut, Garud, Nagarajan, Sabnis, Dzyuba. Contributed reagents and analytic tools: Fudala, I. Gryczynski, Z. Gryczynski, Borejdo.

Wrote or contributed to the writing of the manuscript: Raut, Fudala, Gryczynski, Lacko.

\section{References}

Amar MJ, D’Souza W, Turner S, Demosky S, Sviridov D, Stonik J, Luchoomun J, Voogt J, Hellerstein M, Sviridov D, et al. (2010) 5A apolipoprotein mimetic peptide promotes cholesterol efflux and reduces atherosclerosis in mice. J Pharmacol Exp Ther 334:634-641.

Anantharamaiah G (1986) Synthetic peptide analogs of apolipoproteins, Methods Enzymol 128:627-647.

Anantharamaiah GM, Jones JL, Brouillette CG, Schmidt CF, Chung BH, Hughes TA, Bhown AS, and Segrest JP (1985) Studies of synthetic peptide analogs of the amphipathic helix. Structure of complexes with dimyristoyl phosphatidylcholine. J Biol Chem 260:10248-10255. 
Anantharamaiah GM, Mishra VK, Garber DW, Datta G, Handattu SP, Palgunachari MN, Chaddha M, Navab M, Reddy ST, Segrest JP, et al. (2007) Structural requirements for antioxidative and anti-inflammatory properties of apolipoprotein A-I mimetic peptides. J Lipid Res 48:1915-1923.

Angelin B, Parini P, and Eriksson M (2002) Reverse cholesterol transport in man promotion of fecal steroid excretion by infusion of reconstituted HDL. Atheroscler Suppl 3:23-30.

Barter PJ, Nicholls S, Rye KA, Anantharamaiah GM, Navab M, and Fogelman AM (2004) Antiinflammatory properties of HDL. Circ Res 95:764-772.

Bloedon LT, Dunbar RL, Duffy D, Pinell-Salles P, Navab M, Fogelman A, and Rader DJ (2006) Oral administration of the apolipoprotein A-I mimetic peptide D-4F in humans with CHD improves HDL anti-inflammatory function after a single dose (Abstract). Circulation 114:II_288-II_289.

Bourdi M, Amar M, Remaley AT, and Terse PS (2018) Intravenous toxicity and toxicokinetics of an HDL mimetic, Fx-5A peptide complex, in cynomolgus monkeys. Regul Toxicol Pharmacol 100:59-67.

Bricarello DA, Smilowitz JT, Zivkovic AM, German JB, and Parikh AN (2011) Reconstituted lipoprotein: a versatile class of biologically-inspired nanostructures. ACS Nano 5:42-57.

Calabresi L, Sirtori CR, Paoletti R, and Franceschini G (2006) Recombinant apolipoprotein A-IMilano for the treatment of cardiovascular diseases. Curr Atheroscler Rep 8:163-167.

Chapman MJ (1980) Animal lipoproteins: chemistry, structure, and comparative aspects. J Lipid Res 21:789-853.

Counsell RE and Pohland RC (1982) Lipoproteins as potential site-specific delivery systems for diagnostic and therapeutic agents. J Med Chem 25:1115-1120.

Curtiss LK, Valenta DT, Hime NJ, and Rye KA (2006) What is so special about apolipoprotein AI in reverse cholesterol transport? Arterioscler Thromb Vasc Biol 26:12-19.

Dang CV (2012) Links between metabolism and cancer. Genes Dev 26:877-890.

Dessì S, Batetta B, Pulisci D, Accogli P, Pani P, and Broccia G (1991) Total and HDL cholesterol in human hematologic neoplasms. Int J Hematol 54:483-486.

Febbraio M, Hajjar DP, and Silverstein RL (2001) CD36: a class B scavenger receptor involved in angiogenesis, atherosclerosis, inflammation, and lipid metabolism. $J$ Clin Invest 108:785-791.

Feng H, Wang M, Wu C, Yu J, Wang D, Ma J, and Han J (2018) High scavenger receptor class $\mathrm{B}$ type I expression is related to tumor aggressiveness and poor prognosis in lung adenocarcinoma: a STROBE compliant article. Medicine (Baltimore) 97:e0203.

Frank PG and Marcel YL (2000) Apolipoprotein A-I: structure-function relationships. J Lipid Res 41:853-872.

Gallois L, Fiallo M, and Garnier-Suillerot A (1998) Comparison of the interaction of doxorubicin, daunorubicin, idarubicin and idarubicinol with large unilamellar vesicles. Circular dichroism study. Biochim Biophys Acta 1370:31-40.

Gallois L, Fiallo M, Laigle A, Priebe W, and Garnier-Suillerot A (1996) The overall partitioning of anthracyclines into phosphatidyl-containing model membranes depends neither on the drug charge nor the presence of anionic phospholipids. Eur $J$ Biochem 241:879-887.

Kökoğlu E, Karaarslan I, Karaarslan HM, and Baloğlu H (1994) Alterations of serum lipids and lipoproteins in breast cancer. Cancer Lett 82:175-178.

Kuai R, Li D, Chen YE, Moon JJ, and Schwendeman A (2016) High-density lipoproteins: nature's multifunctional nanoparticles. ACS Nano 10:3015-3041.

Kuai R, Subramanian C, White PT, Timmermann BN, Moon JJ, Cohen MS, and Schwendeman A (2017) Synthetic high-density lipoprotein nanodisks for targeted withalongolide delivery to adrenocortical carcinoma. Int J Nanomedicine 12 $6581-6594$

Lacko AG and Remaley AT (2016) inventors, US Department of Health and Human Services (HHS), University of North Texas Health Science Center, assignee. Drug delivery vehicle. US20150343069A1.

Lacko AG, Sabnis NA, Nagarajan B, and McConathy WJ (2015) HDL as a drug and nucleic acid delivery vehicle. Front Pharmacol 6:247.

Lee H, Jeong CW, Kwak C, Kim HH, Seo SI, Lee HM, Oh JJ, Lee SC, Hong SK, Lee SE, et al. (2017) Preoperative cholesterol level is associated with worse pathological outcomes and postoperative survival in localized renal cell carcinoma patients: a propensity score-matched study. Clin Genitourin Cancer 15:e935-e941.

Lentz BR (1989) Membrane "fluidity" as detected by diphenylhexatriene probes. Chem Phys Lipids 50:171-190.

Li J, Wang J, Li M, Yin L, Li XA, and Zhang TG (2016) Up-regulated expression of scavenger receptor class B type 1 (SR-B1) is associated with malignant behaviors and poor prognosis of breast cancer. Pathol Res Pract 212:555-559.

Levy RI (1981) Cholesterol, lipoproteins, apoproteins, and heart disease: present status and future prospects. Clin Chem 27:653-662.

Lorusso D, Di Stefano A, Carone V, Fagotti A, Pisconti S, and Scambia G (2007) Pegylated liposomal doxorubicin-related palmar-plantar erythrodysesthesia ('hand-foot' syndrome). Ann Oncol 18:1159-1164.
Muntoni S, Atzori L, Mereu R, Satta G, Macis MD, Congia M, Tedde A, Desogus A, and Muntoni S (2009) Serum lipoproteins and cancer. Nutr Metab Cardiovasc Dis 19:218-225.

Navab M, Anantharamaiah GM, Reddy ST, Van Lenten BJ, Datta G, Garber D, and Fogelman AM (2004) Human apolipoprotein A-I and A-I mimetic peptides: potential for atherosclerosis reversal. Curr Opin Lipidol 15:645-649.

Panchoo M and Lacko A (2018) Scavenger receptor class B type 1 regulates neuroblastoma cell proliferation, migration and invasion. Biochem Biophys Res Commun 495:614-620.

Rajora MA and Zheng G (2016) Targeting SR-BI for cancer diagnostics, imaging and therapy. Front Pharmacol 7:326.

Raut S, Dasseux JL, Sabnis NA, Mooberry L, and Lacko A (2018a) Lipoproteins for therapeutic delivery: recent advances and future opportunities. Ther Deliv 9: 257-268.

Raut S, Mooberry L, Sabnis N, Garud A, Dossou AS, and Lacko A (2018b) Reconstituted HDL: drug delivery platform for overcoming biological barriers to cancer therapy. Front Pharmacol 9:1154

Roy A, Kucukural A, and Zhang Y (2010) I-TASSER: a unified platform for automated protein structure and function prediction. Nat Protoc 5:725-738.

Rye KA, Bursill CA, Lambert G, Tabet F, and Barter PJ (2009) The metabolism and anti-atherogenic properties of HDL. J Lipid Res $\mathbf{5 0}$ (Suppl):S195-S200.

Sabnis N, Nair M, Israel M, McConathy WJ, and Lacko AG (2012) Enhanced solubility and functionality of valrubicin (AD-32) against cancer cells upon encapsulation into biocompatible nanoparticles. Int J Nanomedicine 7:975-983.

Santos CR and Schulze A (2012) Lipid metabolism in cancer. FEBS J 279:2610-2623.

Shahzad MM, Mangala LS, Han HD, Lu C, Bottsford-Miller J, Nishimura M, Mora EM, Lee JW, Stone RL, Pecot CV, et al. (2011) Targeted delivery of small interfering RNA using reconstituted high-density lipoprotein nanoparticles. Neoplasia 13:309-319.

Soran H, Schofield JD, and Durrington PN (2015) Antioxidant properties of HDL. Front Pharmacol 6:222.

Sreerama N and Woody RW (2000) Estimation of protein secondary structure from circular dichroism spectra: comparison of CONTIN, SELCON, and CDSSTR methods with an expanded reference set. Anal Biochem 287:252-260.

Sviridov DO, Andrianov AM, Anishchenko IV, Stonik JA, Amar MJ, Turner S, and Remaley AT (2013) Hydrophobic amino acids in the hinge region of the 5A apolipoprotein mimetic peptide are essential for promoting cholesterol efflux by the ABCA1 transporter. J Pharmacol Exp Ther 344:50-58.

Tall AR and Small DM (1978) Plasma high-density lipoproteins. N Engl J Med 299: 1232-1236.

Thaxton CS, Rink JS, Naha PC, and Cormode DP (2016) Lipoproteins and lipoprotein mimetics for imaging and drug delivery. Adv Drug Deliv Rev 106 (Pt A):116-131.

Twiddy AL, Cox ME, and Wasan KM (2012) Knockdown of scavenger receptor class B type I reduces prostate specific antigen secretion and viability of prostate cancer cells. Prostate 72:955-965.

von Eckardstein A, Nofer JR, and Assmann G (2001) High density lipoproteins and arteriosclerosis. Role of cholesterol efflux and reverse cholesterol transport. Arterioscler Thromb Vasc Biol 21:13-27.

Whitmore L and Wallace BA (2004) DICHROWEB, an online server for protein secondary structure analyses from circular dichroism spectroscopic data. Nucleic Acids Res 32:W668-W673.

Whitmore L and Wallace BA (2008) Protein secondary structure analyses from circular dichroism spectroscopy: methods and reference databases. Biopolymers 89: 392-400.

Yang J, Yan R, Roy A, Xu D, Poisson J, and Zhang Y (2015) The I-TASSER Suite: protein structure and function prediction. Nat Methods 12:7-8.

Yuan B, Wu C, Wang X, Wang D, Liu H, Guo L, Li XA, Han J, and Feng H (2016) High scavenger receptor class B type I expression is related to tumor aggressiveness and poor prognosis in breast cancer. Tumour Biol 37:3581-3588.

Zamanian-Daryoush M and DiDonato JA (2015) Apolipoprotein A-I and cancer. Front Pharmacol 6:265.

Zelent B, Kuśba J, Gryczynski I, Johnson ML, and Lakowicz JR (1996) Distancedependent fluorescence quenching of p-bis [2-(5-phenyloxazolyl)] benzene by various quenchers. J Phys Chem 100:18592-18602.

Zhang Y, Zanotti I, Reilly MP, Glick JM, Rothblat GH, and Rader DJ (2003) Overexpression of apolipoprotein A-I promotes reverse transport of cholesterol from macrophages to feces in vivo. Circulation 108:661-663.

Address correspondence to: Sangram Raut, Department of Physiology and Anatomy, UNT Health Science Center, 3500 Camp Bowie Blvd, Fort Worth, TX 76107. Dr. Andras Lacko, Department of Physiology and Anatomy, UNT Health Science Center, 3500 Camp Bowie Blvd, Fort Worth, TX 76107. E-mail: andras.lacko@unthsc.edu 